\title{
Characterization and outcomes of acute myocardial injury in COVID-19 intensive care patients
}

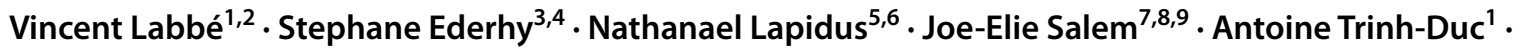 \\ Ariel Cohen ${ }^{3,4,10}$. Muriel Fartoukh ${ }^{1,2}$. Guillaume Voiriot ${ }^{1,2}$
}

Received: 21 July 2020 / Accepted: 21 November 2020 / Published online: 3 January 2021

(c) Springer-Verlag GmbH Germany, part of Springer Nature 2021

\begin{tabular}{ll}
\multicolumn{2}{l}{ Abbreviations } \\
cTn & Cardiac troponin \\
BNP & B-type natriuretic peptide \\
COVID-19 & Coronavirus Disease 2019 \\
ECG & Electrocardiography \\
Hs-cTnI & High sensitivity cardiac troponin I \\
ICU & Intensive care unit \\
MI & Myocardial injury \\
TTE & Two-dimensional transthoracic \\
& echocardiography
\end{tabular}

Acute myocardial injury (MI), defined by an increase in cardiac troponin (cTn), is common in patients hospitalized with COVID-19. MI has been associated with mortality in series mostly retrospective, including heterogeneous COVID-19 patients presenting with mild to critically ill conditions [1, 2]. Measurements of cardiac troponin (cTn) were often obtained only at baseline and requested based on clinical judgment. Thus, the prevalence of MI in the intensive care unit (ICU) patients evaluated by systematic serial

Vincent Labbé

vincent.labbe@aphp.fr

1 Sorbonne Université, Assistance Publique-Hôpitaux de Paris (AP-HP), Service de Médecine Intensive Réanimation, Département Médico-Universitaire APPROCHES, Hôpital Tenon, Paris, France

2 Université Paris Est Créteil, Groupe de Recherche Clinique CARMAS, Créteil, France

3 Department of Cardiology, UNICO-GRECO Cardio-Oncology Program, Hôpital Saint-Antoine, Assistance Publique-Hôpitaux de Paris (AP-HP), Paris, France

4 INSERM U 856, 75013 Paris, France

5 Sorbonne Université, INSERM, Institut Pierre Louis d'Epidémiologie et de Santé Publique IPLESP, Assistance Publique-Hôpitaux de Paris (AP-HP), Paris, France
cTn assessments is unknown. In addition, information for comprehension of the potential underlying mechanism leading to MI is still lacking. In this series of consecutive ICU COVID-19 patients, MI was assessed by a comprehensive workup including sequential cTn dosages, electrocardiography (ECG), and two-dimensional transthoracic echocardiography (TTE) to address the question of its prevalence, characterization, and prognostic value in the ICU setting.

All the consecutive patients with laboratory-confirmed SARS-CoV-2 infection admitted to our dedicated COVID19 ICU between February 22 and April 31, 2020, were analysed. Laboratory confirmation of SARS-CoV-2 was defined as a positive result of real-time RT-PCR assay of nasal and pharyngeal swabs. The study was approved by the local Institutional Ethical Board (Sorbonne University, CER-2020-14). Cardiac investigations were systematically collected including daily dosage of High sensitivity cardiac troponin I (Hs-cTnI) during the first week of ICU stay, and B-type natriuretic peptide (BNP), ECG, and a TTE on ICU admission. The presence of MI was defined by the highest Hs-cTnI value above the 99th percentile upper reference limit and a change in values of $\geq 20 \%$ within the first $48 \mathrm{~h}$ (Hs-cTnI initial value) [3]. Studied outcomes were the

6 Sorbonne University, Assistance Publique-Hôpitaux de Paris (AP-HP), Public Health Department, Saint-Antoine Hospital, 75012 Paris, France

7 Sorbonne University, Assistance Publique-Hôpitaux de Paris (AP-HP), Department of Pharmacology, Pitié-Salpêtrière Hospital, 75013 Paris, France

8 Sorbonne University, Assistance Publique-Hôpitaux de Paris (AP-HP), Department of Cardiology, Pitié-Salpêtrière Hospital, 75013 Paris, France

9 Sorbonne University, UNICO-GRECO Cardio-Oncology Program, CIC-1901, INSERM, 75013 Paris, France

10 Sorbonne Université, UMR-S ICAN 1166, Paris, France 
Table 1 Characteristics, management, and outcome at day 28 of patients with COVID-19

Total, $n=92^{\mathrm{a}}$

No acute myocar- Acute Myocardial $p$ dial injury, $n=39 \quad$ injury, $n=53$

\section{Baseline characteristics}

Age, median (IQR), years

Male, No. (\%)

Body mass index, median (IQR), $\mathrm{kg} / \mathrm{m}^{2}$

Time to ICU admission from symptoms onset, median (IQR), days

Direct ICU admission (from home or ED), No. (\%)

Active smoking, No. (\%)

Diabetes mellitus, No. (\%)

Arterial hypertension, No. (\%)

Coronary artery disease, No. (\%)

Coronary revascularization ${ }^{\mathrm{b}}$, No. (\%)

Congestive heart disease, No. (\%)

Left-sided significant valve disease, No. (\%)

ACEI/ARB, No. (\%)

\section{Acute myocardial injury characteristics}

Electrocardiography on ICU admission

Heart rate, median (IQR), beats/min

ST segment elevation, No. (\%)

ST segment depression, No. (\%)

$\mathrm{T}$ wave inversion, No. (\%)

Laboratory findings on ICU admission, median (IQR) ${ }^{\mathrm{c}}$

Hs-cTnI ${ }^{\mathrm{d}}, \mathrm{pg} / \mathrm{mL}$

$\mathrm{BNP}, \mathrm{pg} / \mathrm{mL}$

Creatinine, micromol/L

C-reactive protein, $\mathrm{mg} / \mathrm{L}$

Leukocytes, $\mathrm{G} / \mathrm{L}^{-1}$

Initial transthoracic echocardiography ${ }^{\mathrm{e}}$, No. (\%)

Left ventricular ejection fraction, median (IQR), \%

Normal (LVEF $\geq 50 \%$ ), No. (\%)

Mildly to Moderately impaired (LVEF 49-31\%), No. (\%)

Severely impaired ( $\leq 30 \%)$, No. $(\%)$

Right ventricular severe dilatation, No. $(\%)^{\mathrm{f}}$

Pericardial effusion, No. (\%) ${ }^{\mathrm{g}}$

\section{Initial severity and organ failure management}

SAPS II score, median (IQR)

SOFA score, median (IQR)

Catecholamine, No. (\%)

Invasive mechanical ventilation, No. (\%)

Renal replacement therapy, No. (\%)

$\mathrm{ECMO}^{\mathrm{h}}$, No. $(\%)$

\section{Outcomes at day-28}

Cardio-vascular event, No. (\%)

Death from any cause, No. (\%)

Cardiac arrest from cardiogenic origin, No. (\%)

Cardiogenic shock ${ }^{\mathrm{i}}$, No. (\%)

Arterial thrombotic event $\mathrm{t}^{\mathrm{j}}$, No. (\%)

ICU-free days ${ }^{\mathrm{k}}$, median (IQR), days

$\begin{array}{cl}62(53-69) & 60(52-65) \\ 72(78.3) & 29(74.4) \\ 28(24-31) & 28(24-31) \\ 8(6-11) & 8(6-13) \\ 49(53.3) & 17(43.6) \\ 6(6.5) & 3(7.7) \\ 24(26.1) & 12(30.8) \\ 58(63.0) & 18(46.2) \\ 16(17.4) & 4(10.3) \\ 10(10.9) & 3(7.7) \\ 8(8.7) & 2(5.1) \\ 3(3.3) & 0(0) \\ 42(45.7) & 12(30.8)\end{array}$

$63(55-71)$

0.07

$43(81.1)$

0.45

$27(25-31)$

0.72

$8(5-10)$

0.14

$32(60.4)$

$3(5.7)$

0.14

0.69

$12(22.6)$

0.47

40 (75.5)

0.005

$12(22.6)$

0.17

7 (13.2)

0.51

$6(11.3)$

3 (5.7)

30 (56.6)

0.46

0.26

0.02

\begin{tabular}{|c|c|c|c|}
\hline $87(75-100)$ & $90(77-100)$ & $81(75-100)$ & 0.40 \\
\hline $1(1.1)$ & $0(0.0)$ & $1(1.9)$ & 1.00 \\
\hline $3(3.3)$ & $1(2.6)$ & $2(3.8)$ & 1.00 \\
\hline $14(15.4)$ & $1(2.6)$ & $13(24.5)$ & 0.01 \\
\hline $33(15-119)$ & $12(7-18)$ & $112(54-260)$ & $<0.001$ \\
\hline $37(18-116)$ & $20(13-59)$ & $78(25-188)$ & $<0.001$ \\
\hline $80(67-128)$ & $74(60-82)$ & $102(70-199)$ & $<0.001$ \\
\hline $190(117-271)$ & 180 (116-249) & $192(121-275)$ & 0.70 \\
\hline $3.43(6.06$ 11.06) & $8.80(5.97-12.79)$ & $8.16(6.65-10.34)$ & 0.25 \\
\hline $60(50-60)$ & $60(55-60)$ & $55(50-60)$ & 0.02 \\
\hline $74(87.1)$ & $33(94.3)$ & $41(82)$ & 0.11 \\
\hline $7(8.2)$ & $2(5.7)$ & $5(10)$ & 0.69 \\
\hline $4(4.7)$ & 0 & $4(8)$ & 0.14 \\
\hline $2(2.4)$ & $0(0.0)$ & $2(4.1)$ & 0.51 \\
\hline $16(20.0)$ & $4(11.4)$ & $12(26.7)$ & 0.16 \\
\hline $40(34-49)$ & $37(28-41)$ & $45(37-54)$ & $<0.001$ \\
\hline $7(4-8)$ & $5(3-7)$ & $8(6-10)$ & $<0.001$ \\
\hline $59(64.1)$ & $20(51.3)$ & $39(73.6)$ & 0.05 \\
\hline $83(90.2)$ & $31(79.5)$ & $52(98.1)$ & 0.004 \\
\hline $23(25.0)$ & $5(12.8)$ & $18(34.0)$ & 0.03 \\
\hline $6(6.5)$ & $2(5.1)$ & $4(7.5)$ & 1.00 \\
\hline $23(25.0)$ & $4(10.3)$ & $19(35.8)$ & 0.01 \\
\hline $18(19.6)$ & $4(10.3)$ & $14(26.4)$ & 0.07 \\
\hline $1(1.1)$ & $0(0.0)$ & $1(1.9)$ & 1.00 \\
\hline $4(4.3)$ & $0(0.0)$ & $4(7.5)$ & 0.13 \\
\hline $4(4.3)$ & $0(0.0)$ & $4(7.5)$ & 0.13 \\
\hline $0(0-15)$ & $5(0-20)$ & $0(0-8)$ & 0.03 \\
\hline
\end{tabular}

Characteristics of patients are compared with Mann-Whitney-Wilcoxon tests for quantitative variables and Fisher's exact test for categorical variables, according to acute myocardial injury 
Table 1 (continued)

$\overline{A C E I}$ angiotensin-converting enzyme inhibitor, $A R B$ angiotensin receptor blocker, $B N P$ binding natriuretic protein, $E D$ emergency department, $H s$-cTnI high sensitivity cardiac troponin I, ICU intensive care unit, $L V E F$ left ventricular ejection fraction, SAPS Simplified Acute Physiology Score, SOFA Sepsis-related Organ Failure Assessment, $V v / V a-E C M O$ venovenous/venoarterial extracorporeal membrane oxygenation;

${ }^{a}$ Missing data: Time to ICU admission from symptoms onset for 1 patient, Electrocardiography for 1 patient, B-type natriuretic peptide for 4 patients, left ventricular systolic ejection fraction for 7 patients, right ventricular dilatation for 8 patients, pericardial effusion for 12 patients

${ }^{\mathrm{b}}$ Percutaneous coronary intervention, $n=9$; Coronary Artery Bypass Graft Surgery, $n=3$

${ }^{\mathrm{c}}$ Highest values during the first $48 \mathrm{~h}$ from intensive care unit admission;

${ }^{\mathrm{d}}$ Measured with the Abbott Architect Method (Abbott, Lake Forest, IL 60,045, USA) wherein the 99th percentile for a normal population is $26 \mathrm{ng} / \mathrm{ml}[3]$

${ }^{\mathrm{e}}$ Median (IQR) time from ICU admission: $0(0-1)$ day; performed by trained operators with competence in advanced critical care TTE

${ }^{\mathrm{f}}$ Right ventricular severe dilatation: end-diastolic area ratio $\geq 1$; paradoxical interventricular septum, $n=0$

${ }^{\mathrm{g}}$ Pericardial tamponade, $n=0$

${ }^{\mathrm{h}} \mathrm{Vv}$-ECMO, $n=5$; Va-ECMO, $n=1$ (in the acute myocardial injury group)

${ }^{\mathrm{i}}$ Patients fulfilled the following criteria: mean atrial pressure $<65 \mathrm{~mm} \mathrm{Hg}$ without a vasopressor agent or need for vasopressor therapy to correct hypotension, low cardiac output, left ventricular systolic dysfunction without inotrope support; elevation of left heart pressures, at least one evidence of tissue hypo-perfusion

${ }^{\mathrm{j}}$ Ischaemic stroke, $n=3$; non-cerebrovascular thromboembolism, $n=1$

${ }^{\mathrm{k}}$ Calculated as 28 minus the length of ICU stay

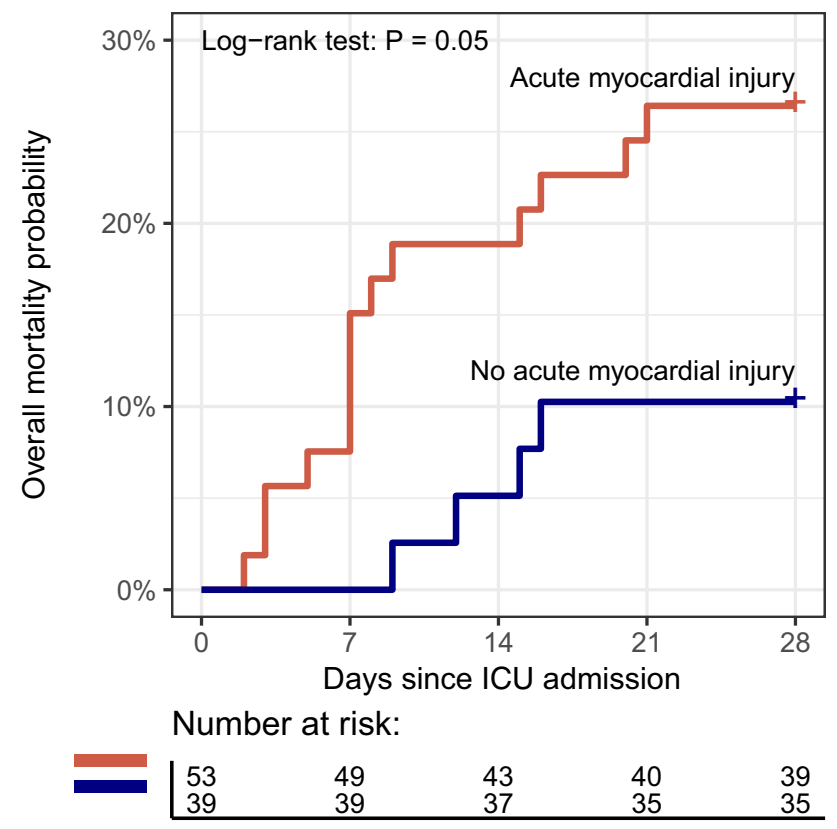

Fig. 1 Kaplan-Meier curves for overall mortality at day 28 as a function of the presence of an acute myocardial injury (log-rank test: $p=0.05)$. ICU, intensive care unit

overall mortality at day- 28 and the incidence of cardiovascular events (a composite of death, cardiac-arrest, cardiogenic shock, and arterial thrombotic event) at day 28 . The association between MI and outcomes was estimated by logistic regression.

Overall, 92 patients (78.3\% men; age 62 [53-69] years) were analysed (Table 1). COVID-19 was diagnosed in ambulant setting, hospital (emergency department or conventional

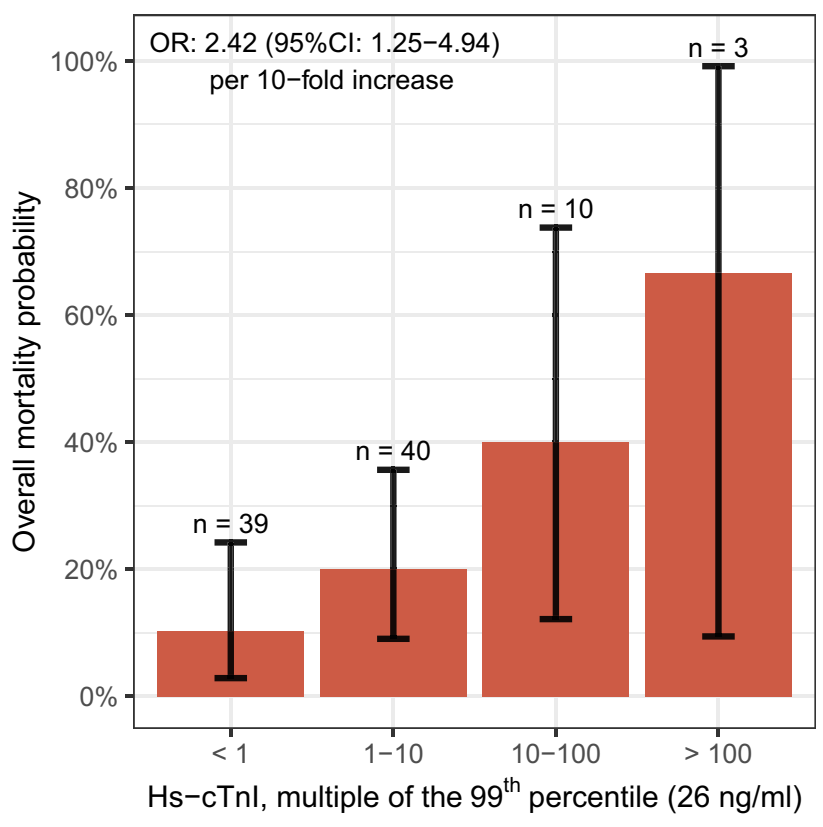

Fig. 2 Probability of overall mortality at day 28 as a function of High sensitivity cardiac Troponin-I initial ${ }^{\text {a }}$ values. $H s-c T n I$ high sensitivity cardiac troponin I, ICU intensive care unit. The bars represent the proportions of patients who died up to day 28 with their $95 \%$ confidence interval, according to their Hs-cTnI on ICU admission. Patient count in each Hs-cTnI class is reported above bars. ${ }^{a}$ Highest values during the first $48 \mathrm{~h}$ of ICU admission

wards), and ICU in respectively 4 (4.3\%), 70 (76.1\%), and 18 (19.6\%) patients. MI was diagnosed in 53 patients $(57.6 \%$; 95\% confidence interval[CI], 46.8-67.9\%) with a Hs-cTnI initial value of 112 (54-260) $\mathrm{pg} / \mathrm{ml}$. Among patient with MI, $13(24.5 \%)$ presented ECG abnormalities including $1(1.9 \%)$ 
ST-segment elevation, 2 (3.8\%) ST-segment depression and $13(24.5 \%) \mathrm{T}$ wave inversion. Patients with MI had higher BNP levels (78 [20-188] vs. 20 [13-59], $p<0.001)$ and a lower left ventricular ejection fraction (55 [50-60] vs. 60 [55-60], $p=0.02$ ) than patients without MI. A greater proportion of patients with MI required catecholamine, invasive mechanical ventilation, and renal replacement therapy. Cardiovascular events occurred in 23 (25\%) patients, including cardiac arrest $(n=1,1.1 \%)$, cardiogenic shock $(n=4,4.3 \%)$, arterial thrombotic event $(n=4,4.3 \%)$, and death $(n=18$; $19.6 \%$ ). Figure 1 illustrates the association of mortality with MI (Kaplan-Meier survival curves, log-rank test $p=0.05$ ). At day 28, the Odds Ratio (OR) for death and cardiovascular events in patients with versus without MI were 3.14 (95\% CI 1.02-11.89) and 4.22 (95\%CI 1.43-12.40), respectively. When adjusting on sepsis-related organ failure assessment, these associations were not significant (OR 1.74, 95\% CI 0.49-7.09 and OR 2.01, 95\%CI 0.56-8.31, respectively). The magnitude of the Hs-cTnI initial values was associated with overall mortality (crude [OR] 2.42; 95\% CI 1.25-4.94 per tenfold increase; Fig. 2). Median daily Hs-cTnI values during the first week of ICU admission remained higher in non-survivors, as compared with survivors (see Figure E1 in the online supplement data).

In this cohort of consecutive critically ill COVID19 patients, the prevalence of MI was higher than that reported in non-ICU patients, suggesting that MI is related to an overall severity and a poor prognosis $[1,2]$. Despite its association with an increased BNP level and a decreased left ventricular ejection fraction, MI rarely induced severe left ventricular systolic dysfunction. Severe right ventricular dilatation was also rarely diagnosed in our cohort, despite severe acute respiratory disease requiring mechanical ventilation. In line with our results, an international survey in COVID 19 patients reported left and right ventricular severe impairment in only $9 \%$ and $6 \%$ of cases [4]. As suggested by the absence of ECG abnormalities in most of our patients, MI may be mediated through non-ischemic mechanisms, such as cytokine storm or direct entry of SARS-CoV-2 into myocardial cells [5]. However, coronary mechanisms like microvascular damage, supply-demand inequity, or destabilization of atheroma cannot be excluded $[2,5]$.

To summarize, acute myocardial injury is very frequent in critically ill COVID-19 patients and is associated with severity.

Supplementary Information The online version contains supplementary material available at https://doi.org/10.1007/s15010-020-01560-y.
Author contributions VL, SE, MF, and GV contributed to study conception and design. VL, SE, GV, AT participated in acquiring the data. VL, AC, GV, SE, NL, and MF analyzed and interpreted the study data. VL drafted the original manuscript. All authors revised the manuscript for important intellectual content. All authors read and approved the final manuscript.

Funding No source of funding.

Availability of data and materials All data and materials are fully complying with field standards and might be available after request.

\section{Compliance with ethical standards}

Conflict of interest $\mathrm{AC}$ received a research grant from Resicard; and consultant/advisory board fees from Amgen, AstraZeneca, Bayer, Bristol-Myers Squibb, Boehringer Ingelheim, Novartis, and Pfizer, unrelated with the present study. GV received research grant from BioMérieux, SOS Oxygène, Janssen unrelated to the present study; and advisory board fees from BioMérieux unrelated to the present study. VL receives advisory board fees from Amomed unrelated with the present study. AT, JS, MF, NL, and SE declared no relevant conflict of interest.

Ethics approval The study has been approved by the local institutional ethical board (Sorbonne University, CER-2020-14) as a component of standard care and patient consent was waived, as per French Law.

Consent to participate Written and oral information was given to the patient or next of kin.

\section{References}

1. Santoso A, Pranata R, Wibowo A, Al-Farabi MJ, Huang I, Antariksa B. Cardiac injury is associated with mortality and critically ill pneumonia in COVID-19: a meta-analysis. Am J Emerg Med. 2020;S0735-6757:30280-1.

2. Lala A, Johnson KW, Januzzi JL, Russak AJ, Paranjpe I, Richter $\mathrm{F}$, et al. Prevalence and impact of myocardial injury in patients hospitalized with COVID-19 infection. J Am Coll Cardiol. 2020;S0735-1097:35552-62.

3. Kozinski M, Krintus M, Kubica J, Sypniewska G. High-sensitivity cardiac troponin assays: from improved analytical performance to enhanced risk stratification. Crit Rev Clin Lab Sci. 2017;54:143-72.

4. Dweck MR, Bularga A, Hahn RT, Bing R, Lee KK, Chapman $\mathrm{AR}$, et al. Global evaluation of echocardiography in patients with COVID-19. Eur Heart J Cardiovasc Imaging. 2020;1-10

5. Clerkin KJ, Fried JA, Raikhelkar J, Sayer G, Griffin JM, Masoumi A, et al. COVID-19 and cardiovascular disease. Circulation. 2020;141:1648-55. 